\title{
LES PROTEINES MAJEURES DE LA SECRETION PROSTATIQUE
}

\author{
Jean Y. Dubé*, Ph.D., Laboratoire de Biorégulation hormonale, \\ Centre Hospitalier de l'Université Laval, 2705, boul. Laurier \\ Ste-Foy, Québec, Canada, G1V 4G2 \\ *Professeur titulaire, Dépt. de médecine, Université Laval, Ste Foy, Québec, Canada
}

\section{MAJOR PROSTATIC SECRETORY PROTEINS.}

Human prostatic secretion contains only a few major proteins among which one finds albumin, acid phosphatase, prostate specific antigen (PSA), prostatic secretory protein of 94 amino acids (PSP94), Zn- $\alpha_{2}$-glycoprotein and $\alpha_{1}$-acid glycoprotein. The roles of most of these proteins are not entirely known. However, PSA has been shown to contribute to the hydrolysis of the seminal coagulum proteins. Probable roles for the other components include modifications of the properties of spermatozoa surface and control of inflammatory responses in male and female urogenital tracts. Keys words : prostatic fluid, ejaculate, seminal plasma, seminal vesicles. Andrologie, 1991, 1 : 56-58.

Bien que les biochimistes se soient intéressés au plasma séminal et au fluide prostatique depuis de très nombreuses années, on savait réellement peu de choses jusqu'à récemment sur la nature même de la plupart des protéines de ce fluide. Avec avènement des techniques modernes de séparation, de purification et de séquençage des protéines, nos connaissances ont progressé rapidement mais demeurent encore très fragmentaires.

La méthode de l'éjaculation fractionnée permet d'obtenir facilement dans les premières gouttes émises un fluide assez représentatif de la sécrétion prostatique puisquil est peu contaminé par la sécrétion vésiculaire et par le sérum. Lorsque les protéines de ce fluide sont analysées par électrophorèse sur gel de polyacrylamide à deux dimensions (Fig. 1), on constate que les protéines majeures sont en nombre beaucoup plus limité que dans le sérum. On sait maintenant que les six protéines principales par ordre de poids moléculaire décroissant sont l'albumine, la phosphatase acide prostatique (PAP), l' $\alpha_{1}$-acide glycoprotéine, la $\mathrm{Zn}$ - $\alpha_{2}$-glycoprotéine, l'antigène spécifique à la prostate (en anglais prostate specific antigen : PSA) et la protéine de sécrétion prostatique de 94 acides aminés (PSP94). Le fluide prostatique contient également une multitude d'autres protéines mineures dont on peut retrouver une liste assez exhaustive dans la revue de Lizana et Eneroth (13). Pour les besoins de cet article, je limiterai mon propos à la biochimie et à la physiologie des six protéines majeures énumérées plus haut.

\section{L'albumine}

L'albumine est un constituant relativement important du fluide prostatique $(0,5-1 \mathrm{~g} / \mathrm{l})$. Elle n'est cependant pas synthétisée par les cellules prostatiques mais elle dérive du sérum par transudation à partir des espaces intercellulaires. On a émis l'hypothèse que le rôle de l'albumine dans le plasma séminal serait d'améliorer la motilité et la survie des spermatozoïdes. Bien que cette hypothèse soit plausible, on n'a pas observé de différences significatives dans les niveaux d'albumine du plasma séminal des hommes fertiles ou infertiles (13).

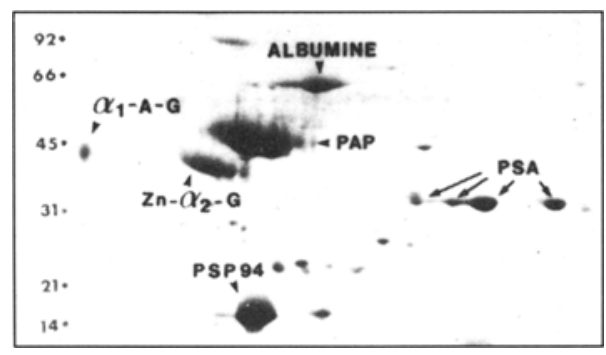

Figure 1: Séparation par électrophorèse sur gel de polyacrylamide à 2 dimensions des protéines du fluide prostatique obtenu par éjaculation fractionnée. Les protéines sont séparées selon leur point isoélectrique (acide à gauche et basique à droite) et selon leur poids moléculaire (dimension verticale). La position où migrent 6 protéines standards de poids moléculaire connu $(14,000$ à 92,000 ) est indiquée à gauche du gel. Les protéines ont été colorées au bleu de Coomassie. Abréviations : $\alpha_{1}$-a-G $=\alpha_{1}$-acide-glycoprotéine ; $\mathrm{Zn}-\alpha_{2}-\mathrm{G}=\mathrm{Zn}-\alpha_{2^{-}}$ glycoprotéine.

\section{La phosphatase acide prostatique}

La PAP est le marqueur du cancer de la prostate utilisé depuis plus de 50 ans. Elle est la protéine la plus abondante du fluide prostatique où elle représente environ $25 \%$ des protéines totales. C'est une sialoglycoprotéine de 100,000 $\mathrm{kDa}$ avec deux sousunités identiques de 50,000 kDa. Sa séquence en acides aminés diffère de celle de la phosphatase acide lysosomiale sur environ $50 \%$ des résidus (17). Le plasma séminal contient quelques substrats potentiels de cette enzyme dont la phosphorylcholine (16). On ne connait pas le rôle physiologique de cette hydrolyse. De plus, la PAP est capable d'hydrolyser le groupement phosphate des protéines phosphorylées sur les résidus tyrosine, sérine et thréonine (12). De telles protéines phosphorylées sont présentes sur la surface du spermatozoïde et on peut penser que leur déphosphorylation, tout comme d'autres modifications des protéines de surface, pourrait jouer un rôle dans le phénomène de la capacitation. Certaines expériences montrent également qu'au moins deux protéines des vésicules séminales, la séménogéline et la fibronectine impliquées dans la formation du coagulum sont phosphorylées $(2,15)$. L'influence de la PAP sur le degré de phosphorylation de ces protéines de même que les conséquences de la présence ou de l'absence des groupements phosphates dans les phénomènes de coagulation et de liquéfaction du sperme restent à déterminer.

L' $\alpha_{1}$-acide glycoprotéine

L' $\alpha_{1}$-acide glycoprotéine est avant tout une protéine sérique synthétisée par le foie. Cependant, nous avons démontré récemment que cette protéine pouvait également être traduite dans la prostate (4). On ne sait pas quelle proportion de la protéine dans le fluide prostatique peut venir du sérum et de la synthèse locale. L' $\alpha_{1}$-acide glycoprotéine a une masse moléculaire de $40 \mathrm{kDa}$ à laquelle les sucres et l'acide sialique contribuent pour environ $50 \%$. Cette protéine possède certaines propriétés immunosuppressives particulièrement lorsqu'elle est déglycosylée (15). Bien qu'on ne possède pas beaucoup de données concrètes pour l'affirmer, l'activité immunosuppressive pourrait servir deux rôles principaux soit ceux de protéger la prostate contre l'inflammation et d'empêcher les spermatozoïdes de provoquer des réactions immunologiques dans le tractus urogénital de la femme.

\section{La $\mathrm{Zn}-\alpha_{2}$-glycoprotéine}

Avec mes collègues, nous avons identifié récemment cette protéine dans la sécrétion prostatique (7). Sa concentration dans le plasma séminal $(0,4 \mathrm{~g} / \mathrm{L})$ est environ 5 fois plus élevée que dans le sérum. On a tout lieu de croire qu'elle est synthétisée en totalité par les cellules prostatiques et qu'elle n'est pas 
concentrée à partir du sérum. La composition en acides aminés de la protéine purifiée du plasma séminal est identique à celle du sérum. Ces résultats indiquent qu'il s'agit bien de la même protéine. Magré son nom, la $\mathrm{Zn}-\alpha_{2^{-}}$ glycoprotéine ne lie pas le zinc qui, comme on le sait, est présent en grande concentration dans le fluide prostatique. Son nom vient plutôt du fait qu'elle a été isolée du sérum par précipitation avec des sels de zinc. La séquence complète des acides aminés de la protéine sérique publiée récemment montre qu'elle a une très grande homologie avec les antigènes d'histocompatibilité et avec une protéine ressemblant à $\mathrm{H}-2$ chez la souris (1). Comme la protéine $\mathrm{H} 2$ est capable de médier une immu-notolérance active, son rôle dans le plasma séminal pourrait donc être semblable à celui de l' $\alpha_{1}$-acide glycoprotéine. Il est intéressant de noter que cette protéine est aussi présente dans les sécrétions apocrines et surtout dans la sueur où elle représente $36 \%$ des protéines totales (15).

\section{L'antigène spécifique à la prostate}

Le PSA a été purifié de tissus prostatiques humains pour la première fois en 1979 (19). Il est devenu rapidement par la suite le marqueur de choix pour le cancer de la prostate. Le PSA est une glycoprotéine de $34 \mathrm{kDa}$ qui comporte trois ou quatre isoformes majeures. La séquence des acides aminés a révélé qu'il s'agissait d'une protéase à sérine apparentée aux kallikréines glandulaires (20). Les seuls substrats connus de cette enzyme sont la séménogéline et la fibronectine (11). Ces deux protéines sécrétées par les vésicules séminales forment la partie solide du coagulum et lors du phénomène de liquéfaction du sperme, elles sont progressivement hydrolysées par le PSA avec ou sans l'aide des multiples autres enzymes protéolytiques contenues dans le plasma séminal. Alors que le coagulum se dissout, les spermatozoïdes deviennent progressivement motiles. Cette motilité accrue pourrait être causée par des peptides libérés pendant l'hydrolyse de la séménogéline (9). Plusieurs observations suggèrent que le zinc pourrait influencer l'hydrolyse de la séménogéline. En effet, nos études ont montré que le zinc se liait fortement à la séménogéline (6). De plus, on sait que le zinc inhibe l'activité enzymatique du PSA (20) et ralentit la liquéfaction du coagulum (10. On peut donc concevoir que lors de l'éjaculation, le zinc prostatique en se fixant à la séménogéline des vésicules séminales enlèverait l'inhibition sur le PSA et lui permettrait d'exercer son activité hydrolytique. Ce construit demeure une hypothèse et d'autres mécanismes de liquéfaction ont également été proposés (14).

\section{La protéine de sécrétion prostatique de 94 acides aminés}

La PSP94 qui est aussi connue sous les noms de $\beta$-inhibine et de $\beta$-microséminoprotéine est une protéine majeure de la sécrétion prostatique et sa concentration dans le plasma séminal est d'environ $1.3 \mathrm{~g} / \mathrm{l}$ (3). C'est une protéine non-glycosylée et qui est absente du sérum. Sa séquence en acides aminés n'indique pas d'homologie globale avec des protéines connues. Cependant, il existe de courtes séquences de la partie carboxyterminale de la protéine qui ont une certaine similarité avec les chaînes A et B de la Binhibine ovarienne (18). On ne croit pas que la PSP94 ait véritablement d'activité de type inhibine in vivo. Alors que l'on pensait que cette protéine était spécifique à la prostate, il a été démontré récemment que l'ARN messager de la PSP94 était exprimé fortement dans quelques tissus tels la muqueuse gastrique et la trachée (18). Ceci suggère qua la PSP94 est reliée aux activités exocrines de certaines cellules épithéliales tout comme la $\mathrm{Zn}-\alpha_{2}$ glycoprotéine. On sait également que la PSP94 est une protéine qui peut se lier aux spermatozoïdes en même temps que d'autres protéines sécrétées par les glandes accessoires mâles. Cette liaison pourrait avoir pour fonction de retarder la capacitation et/ou de masquer des sites antigéniques du spermatozoïde lors de son parcours dans les tractus urogénitaux de l'homme et de la femme. De tels rôles ont également été proposés pour la lactoferrine qui origine des vésicules séminales.

\section{CONCLUSION}

Malgré certains progrès récents dans l'identification des protéines majeures de la sécrétion prostatique, il reste encore beaucoup de points obscurs sur leur rôle exact et en particulier leur interaction avec les spermatozoïdes et leur contribution à la fertilité masculine. De nombreuses études ont tenté d'établir des corrélations entre les concentrations de différents constituants du plasma séminal et certaines caractéristiques du sperme. Dans la majorité des cas, aucune corrélation significative n'a été démontrée. Il faut reconnaître que même chez des individus normaux et fertiles, la concentration des protéines de sécrétion prostatique varie considérablement dans le plasma séminal. Ceci s'explique en partie par les volumes relatifs variables des fluides vésiculaire et prostatique. Cependant, cette variabilité ne signifie pas que la sécrétion prostatique n'a aucun rôle dans la fertilité masculine. D'autres facteurs entrent en ligne de compte. L'un de ceux-ci est la chronologie du contact des spermatozoïdes avec les différentes sécrétions des glandes accessoires mâles. En effet, si les spermatozoïdes viennent en contact avec le fluide vésiculaire avant le fluide prostatique, leur motilité, leur viabilité et leur capacité de décondenser la chromatine en sera sérieusement affectée (5). Ces expériences démontrent que le plasma séminal n'est pas qu'un simple milieu de transport pour les spermatozoïdes et les différentes sécrétions accessoires.

\section{REMERCIEMENTS}

Les travaux de notre Laboratoire cités dans cet article ont été réalisés grâce à des subventions du Conseil de Recherches Médicales du Canada. Nous sommes également reconnaissants envers madame Lucie Turcotte pour son travail dactylographique.

\section{REFERENCES}

1 - Araki T, Gejyo F, Takagaki K, Haupt H, Schwick HC, Burgi W, Marti T, Schaller J, Rickli E, Brossmer R, Atkinson PH, Putnam FW, Schmid K. Complete amino acid sequence of human plasma $\mathrm{Zn}-\alpha$ 2-glycoprotein and its homology to histo compatibility antigens. Proceed. Natl Acad. Sci. (USA), 1988, 85:679-683.

2 - Chantananukul W, Panyim S. In vitro specific phosphorylation of the human seminal proteins. Andrologia, 1982, 14:447-453.

3 - Dubé JY, Frenette G, Paquin R, Chapdelaine P, Tremblay J, Tremblay RR, Lazure C, Seidah N, Chrétien M. Isolation from human seminal plasma of an abundant $16 \mathrm{kDa}$ protein originating from the prostate. Its identification with a 94-residue peptide described as B-inhibin. J. Andrology, 1987, 8:182-189.

4 - Dubé JY, Paradis G, Têtu B, Tremblay RR. Synthesis of $\alpha 1$-acid glycoprotein by the human prostate. Prostate, 1989, 15:251-258.

5 - Eliasson R. Biochemical analysis of semen. Int. J. Androl., 1982, Suppl 5:109-119.

6 - Frenette G, Tremblay RR, Dubé JY. Zinc binding to major human seminal coagulum proteins. Arch. Androl., 1989, 23:155-163.

7 - Frenette G, Dubé JY, Lazure C, Paradis G, Chrétien M, Tremblay RR. The major $40 \mathrm{kDa}$ glycoprotein in human prostatic fluid is identifical to Zn-02-glycoprotein. Prostate, 1987, 11:257-270.

8 - Haagensen DE, Mazoujian G. Biochemistry and immunochemistry of fluid proteins in the breast in gross cystic disease. In "Diseases of the Breast" 3rd edition, Cann C. ed., W.B. Saunders Co, 1986:474-500. 
9- Lilja H, Abrahamsson PA, Lundwall A. Semenogelin, the predominant protein in human semen. Primary structure and identification of closely related proteins in the male accessory sex glands and on the spermatozoa. J. Biol. Chem., 1989, 264:1894-1900.

10 - Lilja H, Laurell CB. Liquefaction of coagulated human semen. Scand. J. Clin. Lab. Invest., 1984, 44:447-452.

11 - Lilja H, Oldbring J, Rannevik G, Laurell CB. Seminal vesicle secreted proteins and their reactions during gelation and liquefaction of human semen. J. Clin. Invest., 1987, 80:281-285.

12 - Lin MF, Clinton GM. Human protastic acid phosphatase has phosphotyrosyl protein phosphatase activity. Biochem. J., 1986, 235:351-357.

13 - Lizana JA, Eneroth P. Proteins in seminal plasma and cervicovaginal secretions. In "Proteins in body fluids, amino acids and tumor markers : diagnostic and clinical aspects", Alan R. Liss Inc., New York, 1983:271-274.

14 - Polak B, Daunter B. Seminal plasma biochemistry. IV : Enzymes involved in the liquefaction of human seminal plasma. Int. J.
Androl., 1989, 12:187-194.

15 - Putnam FW. The plasma proteins. Structure, function, and genetic control. Volume IV, 2nd edition, Academic Press, Orlando, 1984, 420 pp.

16 - Serrano JA, Wasserkrug HL, Serrano AA, Paul BD, Seligman AM. The histochemical demonstration of human prostatic acid phosphatase with phosphorylcholine. Invest. Urol., 1977, 15:123-136.

17 - Sharief FS, Lee H, Leudermann MM, Lundwall A, Deaven LL, Lee CL, Li SSL. Human prostatic acid phosphatase : cDNA cloning, gene mapping and protein sequence homology with lysosomal acid phosphatase. Biochem. Biophys. Res. Commun., 1989, 160:79-86.

18 - Ulvsback $M$, Lindstrom $C$, Weiber $H$, Abrahamsson PA, Lilja H, Lundwall A. Molecular cloning of a small prostate protein known as B microsemino-protein, PSP94 or B inhibin and demonstration of transcripts in non genital tissues. Biochem. Biophys. Res. Commun., 1989, 164:1310-1315.

19 - Wang MC, Valenzuela LA, Murphy GP, Chu TM. Purification of a human prostate specific antigen. Invest. Urol., 1979, 17:159-163.
20 - Watt KWK, Lee PJ, M'Timkulu T, Chan WP, Loor R. Human prostate specific antigen : structural and functional similarity with serine proteases. Proceed. Natl Acad. Sci (USA), 1986, 83:3166-3170.

RESUME : La sécrétion prostatique humaine ne contient que quelques protéines majeures parmi lesquelles on retrouve l'albumine, la phosphatase acide, l'antigène spécifique à la prostate (PSA), la protéine de sécrétion de 94 acides aminés (PSP94), la Zn- 22 glycoprotéine et l' $\alpha 1$-glycoprotéine. Le rôle de la plupart de ces protéines n'est pas entièrement connu. On sait toutefois que le PSA contribue à l'hydrolyse des protéines du coagulum formé lors de l'éjaculation. Les rôles probables des autres constituants incluent l'altération des propriétés de surface du spermatozoïde et le contrôle des réponses inflammatoires dans les tractus urogénitaux de l'homme et de la femme. Mots clefs. fluide prostatique, éjaculat, plasma séminal, vésicules séminales. Andrologie 1991,1 : 56-58. 